AB0831

FREQUENCY OF RENAL CRISIS IN PATIENTS WITH SYSTEMIC SCLEROSIS: NEW DATA FROM A CHINESE COHORT AND A SYSTEMIC REVIEW WITH META-ANALYSIS

D. Xu ${ }^{1}$, J. $\mathrm{He}^{2}, \mathrm{~T} . \mathrm{LI}^{1}, \mathrm{R} . \mathrm{Mu}^{1,1,1}{ }^{1}{ }^{1}$ Peking University Thrid Hospital, Department of Rheumatology and Immunology, Beijing, China; ${ }^{2}$ The Third People's Hospital of Datong, Department of Rheumatology and Immunology, Datong, China

Background: Scleroderma renal crisis (SRC) is a rare but life-threatening complication in SSc, which defined as malignant hypertension and progressive renal failure ${ }^{[1]}$. Previous reports of the prevalence for scleroderma renal crisis (SRC) show variable outcomes.

Objectives: We aim to find out the prevalence for SRC in Chinese, and conduct a meta-analysis to assess the prevalence of SRC in worldwide and investigate possible factors of variability.

Methods: We evaluated the prevalence for SRC and compared the clinical characteristics in SSc patients with or without SRC. A systematic review on the prevalence of SRC was carried out in PubMed, Embase and Cochrane. Meta-analysis was performed using available data on the prevalence for SRC.

Results: 302 patients were enrolled in PKUPH-SSc cohort and the prevalence of SRC was $4.97 \%$. In our cohort, SRC was found in $3.60 \%$ limited cutaneous SSc (IcSSc) and $7.75 \%$ diffused cutaneous SSc (dcSSc) patients. Twenty-nine studies including a total population of 22686 SSc patients were analyzed in the meta-analysis. The overall pooled prevalence of SRC was $4.0 \%$, but heterogeneity among the studies was substantial $\left(I^{2}=86.1 \%, P<0.00001\right)$. Meta-regression revealed that subtype of SSc and geographic factors partially explained this heterogeneity. Sub-group analyses demonstrated that the prevalence of SRC in dcSSc and IcSSc were $9.0 \%$ and $2.0 \%$. The SRC prevalence in SSc was $3.0 \%$ in Asia, $5.0 \%$ in Europe, and $3.0 \%$ in North America, respectively.

Conclusion: Data on our new cohort showed the prevalence of SRC was around $5 \%$ in China, and meta-analysis confirmed that the prevalence of SRC varies among centers. The marked heterogeneity of SRC prevalence was partially explained by subtype of SSc and geographic origin of patients.

REFERENCES:

[1] Woodworth TG, Suliman YA, Li W, Furst DE, Clements P (2016) Scleroderma renal crisis and renal involvement in systemic sclerosis. Nat Rev Nephrol 12 (11):678-91.

Disclosure of Interests: None declared

DOI: 10.1136/annrheumdis-2021-eular.3456

\section{AB0832 PREVALENCE OF OCULAR DISEASES IN PATIENTS WITH SYSTEMIC LUPUS ERYTHEMATOSUS: A RETROSPECTIVE OBSERVATIONAL STUDY IN A TERTIARY HOSPITAL IN TAIWAN}

J. H. Kao ${ }^{1}$, T. Y. Lan ${ }^{1}$, C. H. Lu', C. Y. Shen ${ }^{1}$, K. J. LI' ${ }^{1}$, S. C. Hsieh ${ }^{1} .{ }^{1}$ National Taiwan University Hospital, Department of Internal Medicine, Division of Allergy, Immunology and Rheumatology, Taipei, Taiwan, Republic of China

Background: Systemic lupus erythematosus (SLE) could affect multiple parts of the eye. $(1,2)$ Also, there is risk of ocular toxicity associated with treatment, such as hydroxychloroquine.(3) Early diagnosis is essential to avoid major ocular complications. However, complaints of eye discomfort may be vague, and various presentations may pose challenges on rheumatologists. Knowing the real-world prevalence of ocular problems in patients with SLE is important and helpful, yet this has been less common to be reported.

Objectives: To describe the prevalence of various ophthalmologic diagnoses in patients with SLE.

Methods: This is a retrospective observational study conducted in a tertiary hospital in Taipei, Taiwan. Patients diagnosed with SLE in the period between 1st Jan, 2002 and 31th Dec, 2015 and evaluated by ophthalmologists in 2 years before 29th Jan, 2021 were included. Demographic and clinical data, and ophthalmologic diagnoses were recorded by chart review.

Results: A total of 121 patients were included, and 118 (97.5\%) were female. Average age was 46.2 years [standard deviation (SD) 11.4 years] upon ophthalmologic evaluation, and average duration suffering from lupus was 11.6 years (SD 3.3 years). Keratoconjunctivitis sicca $(n=48,39.7 \%)$, myopia ( $n=39$, $32.2 \%)$, and cataract $(n=21,17.4 \%)$ were the most common findings. It was also noted that suspicious finding of hydroxychloroquine retinal toxicity was found in 12 patients ( $9.9 \%$ of total patients). Also, two patients had lupus retinopathy, and another two were diagnosed with cytomegalovirus retinitis. In addition, glaucoma was diagnosed in 9 patients $(7.4 \%)$, which seemed to be higher than the general population.

Conclusion: Higher prevalence of different ocular problems is noted in this cohort of SLE patients. Keratoconjunctivitis sicca was the most common ocular diagnosis, which is consistent with literature. However, high rates of myopia, cataract, glaucoma, and suspicious hydroxychloroquine retinal toxicity were also found. Above result warrants more aggressive ophthalmologic evaluation in SLE patients. More research on the association of lupus and different ocular diseases is needed in the future.

\section{REFERENCES:}

[1] Silpa-archa S, Lee JJ, Foster CS. Ocular manifestations in systemic lupus erythematosus. Br J Ophthalmol. 2016 Jan;100(1):135-41.

[2] Sivaraj RR, Durrani OM, Denniston AK, Murray PI, Gordon C. Ocular manifestations of systemic lupus erythematosus. Rheumatology (Oxford). 2007 Dec;46(12):1757-62.

[3] Jorge A, Ung C, Young LH, Melles RB, Choi HK. Hydroxychloroquine retinopathy - implications of research advances for rheumatology care. Nat Rev Rheumatol. 2018;14(12):693-703.

Table 1. Demographic data and ophthalmologic diagnosis of patients with systemic lupus erythematosus $(N=121)$

\begin{tabular}{lc}
\hline Variable & $\mathrm{n}(\%$ of N) \\
\hline Age (years) average (SD) & $46.2(11.4)$ \\
Disease duration average (SD) & $11.6(3.3)$ \\
Female sex & $118(97.5)$ \\
Chronic medical disease & \\
Hypertension & $28(23.1)$ \\
Diabetes mellitus & $4(3.3)$ \\
Dyslipidemia & $7(5.8)$ \\
Cancer & $3(2.5)$ \\
End-stage renal disease & $1(0.8)$ \\
Ophthalmologic diagnosis & \\
Keratoconjunctivitis sicca & $48(39.7)$ \\
Myopia & $39(32.2)$ \\
Cataract & $21(17.4)$ \\
Glaucoma & $9(7.4)$ \\
Suspicious retinopathy related to HCQ & $12(9.9)$ \\
Unspecified retinopathy & $5(4.1)$ \\
Lupus retinopathy & $2(1.7)$ \\
CMV retinitis & $2(1.7)$ \\
Central retinal vein thrombosis & $1(0.8)$ \\
Retinal break & $1(0.8)$ \\
Optic neuritis & $1(0.8)$ \\
Vitreal hemornage & $1(0.8)$ \\
Myodesopia & $4(3.3)$ \\
Scleritis & $1(0.8)$ \\
\hline
\end{tabular}

$\mathrm{SD}$, standard deviation; $\mathrm{HCQ}$, hydroxychloroquine; $\mathrm{CMV}$, cytomegalovirus.

Disclosure of Interests: None declared

DOI: 10.1136/annrheumdis-2021-eular.3746

\section{AB0833 HOSPITAL ADMISSIONS FOR RHEUMATOID ARTHRITIS AND OTHER INFLAMMATORY POLYARTHROPATHIES IN THE BRAZILIAN PUBLIC HEALTH SYSTEM}

A. Dal Pra Wendt ${ }^{1}$, F. Milene Cardozo ${ }^{1} .{ }^{1}$ Feevale University, Institute of Health Sciences, Novo Hamburgo, Brazil

Background: Rheumatoid arthritis is an idiopathic autoimmune disease that is characterized by symmetrical peripheral polyarthritis. The disease leads to the destruction of joints due to erosion of both bone and cartilage. The other inflammatory polyarthropathies are a group composed of 61 diseases, according to the International Classification of Diseases (ICD-10). Drug treatment is carried out through outpatient follow-up, with no need for hospitalization in mild and / or controlled cases. When surgical treatment is recommended or there are complications from rheumatoid arthritis or other inflammatory polyarthropathies, hospitalization is indicated. In addition, Brazil has a broad public health system, called the Unified Health System (UHS), responsible for most health procedures carried out at the national level, especially with regard to the socioeconomically vulnerable population.

Objectives: To investigate the number of hospital admissions for rheumatoid arthritis and other inflammatory polyarthropathies in UHS during the past three years.

Methods: This is a quantitative and retrospective survey based on the data available on the website of the Department of Informatics of the Brazilian Unified Health System. The data are freely accessible and public domain. Information was collected about the period between December 2017 and November 2020, referring to the number of hospitalizations for rheumatoid arthritis and other inflammatory polyarthropathies in all five regions that make up Brazil.

Results: The number of hospitalizations for rheumatoid arthritis and other inflammatory polyarthropathies fluctuated, between December 2017 and November 2020 , from 1083 to 1311 per month, with an average of 1184 and a total of 38228 hospitalizations over the three years analyzed. Throughout the national territory, the region with the least monthly admissions was the central-west (which comprises the states of Mato Grosso, Mato Grosso do Sul, Distrito Federal and Goiás), with 2562 total admissions and an average of 71.1 monthly admissions. 
In contrast, the region with the highest number of cases was the southeastern region (where the states of Espírito Santo, Minas Gerais, São Paulo and Rio de Janeiro are located), with a total of 13173 hospitalizations and a monthly average of 365.9 , representing $34,4 \%$ of hospitalizations in the country. Such data is in line with the Brazilian demography, since the central-west region is the second least inhabited (with 16.09 million inhabitants) and the southeastern region is the most densely inhabited (with 80.35 million inhabitants). The number of monthly hospitalizations, which remained above 1000 from the first month analyzed (December 2017) until February 2020, dropped to 990 in March of the same year and 651 in April, maintaining an average of 678.2 in next seven months.

Conclusion: It is concluded that the number of hospitalizations for rheumatoid arthritis and other inflammatory polyarthropathies in Brazil dropped significantly between March and April 2020, a period that coincides with the beginning of the COVID-19 pandemic in the country, possibly as a result both of the changes in the Brazilian medical care model (in relation to the requirements for hospitalization) and as a result of the lower demand by individuals for hospitals due to fear of contamination by the new coronavirus.

REFERENCES:

[1] BATES, B. Propedêutica Médica. 12 ${ }^{\underline{a}}$ ed. Rio de Janeiro: Guanabara Koogan, 2018.

Disclosure of Interests: None declared

DOI: 10.1136/annrheumdis-2021-eular.4053

\section{Validation of outcome measures and biomarkers}

\section{AB0834 DEVELOPMENT OF A CONCEPTUAL FRAMEWORK FOR A PATIENT REPORTED OUTCOME MEASURE TO CAPTURE PATIENTS' PERCEPTIONS OF GLUCOCORTICOID THERAPY DURING TREATMENT FOR RHEUMATIC DISEASES}

S. Bridgewater ${ }^{1,2}$, J. Dawson ${ }^{3}$, M. Ndosi ${ }^{1,2}$, R. J. Black ${ }^{4,5}$, J. T. L. Cheah ${ }^{6}$, E. Dures ${ }^{1,2}$, N. Ghosh ${ }^{7}$, E. A. Hoon ${ }^{8}$, I. Navarro-Millan ${ }^{7}$, D. Pearce-Fisher ${ }^{7}$, P. Richards ${ }^{2}$, C. Ruediger ${ }^{8}$, C. Silverthorne ${ }^{1,2}$, J. Tieu ${ }^{8}$, S. Mackie ${ }^{9,10}$, S. Goodman ${ }^{7}$, C. Hill ${ }^{8}$, J. Robson ${ }^{1,11}$ on behalf of Outcome Measures in Rheumatology (OMERACT) Glucocorticoid Working Group. ${ }^{1}$ University of the West of England - UWE Bristol, Faculty of Health and Applied Sciences, Bristol, United Kingdom; ${ }^{2}$ University Hospitals Bristol and Weston NHS Foundation Trust, Academic Rheumatology, Bristol, United Kingdom; ${ }^{3}$ University of Oxford, Nuffield Department of Population Health, Oxford, United Kingdom; ${ }^{4}$ The University of Adelaide, Discipline of Medicine, Adelaide, Australia; ${ }^{5}$ Royal Adelaide Hospital, Rheumatology Unit, Adelaide, Australia; ${ }^{6}$ University of Massachusetts Medical School, Department of Medicine, Division of Rheumatology, Worcester, United States of America; ${ }^{7}$ Hospital for Special Surgery, Division of Rheumatology, New York, United States of America; ${ }^{8}$ The Queen Elizabeth Hospital, Department of Rheumatology, Adelaide, Australia; ${ }^{9}$ Leeds Teaching Hospitals NHS Trust, Leeds Biomedical Research Centre, Leeds, United Kingdom; ${ }^{10}$ University of Leeds, Institute of Rheumatic and Musculoskeletal Medicine, Leeds, United Kingdom; ${ }^{11}$ University Hospitals Bristol and Weston NHS Foundation Trust, Academic Rheumatology, Bristol, United Kingdom

Background: Glucocorticoids (GCs) are a key treatment for the autoimmune rheumatic diseases; however, they produce numerous physical and psychological side effects. ${ }^{1}$ The Outcome Measures in Rheumatology (OMERACT) Glucocorticoid Working Group have identified that there are no Patient Reported Outcome Measures (PROMs) for assessing the impact of systemic GC therapy across multiple rheumatic diseases from the patient's perspective. ${ }^{2,3}$

Objectives: The aim is to explore the impact of GCs on the symptoms and health-related quality of life of adults with rheumatic inflammatory diseases, to inform items for inclusion in a PROM. Key considerations will include patient perceptions of GC therapy at diagnosis and over the course of treatment, for use in future randomised controlled trials or in clinical practice.

Methods: An international steering committee comprising researchers, rheumatology clinicians, methodologists and patient partners in the UK, Australia and USA developed an initial conceptual framework informed by a review of the literature. Semi-structured interviews were conducted in each country with patients who had an autoimmune rheumatic disease and had received GC therapy. The interviews explored salient aspects of health-related quality of life associated with being treated with GCs.

Results: Interviews have been completed in three continents with patients who had a range of demographic features, rheumatological conditions and duration and dosage of GC therapy. Figure 1 shows the initial conceptual framework for developing the GC PROM (Steroid PRO).

Conclusion: This conceptual framework will act as an evolving guide in the development of a PROM for assessing patients' perspectives of systemic
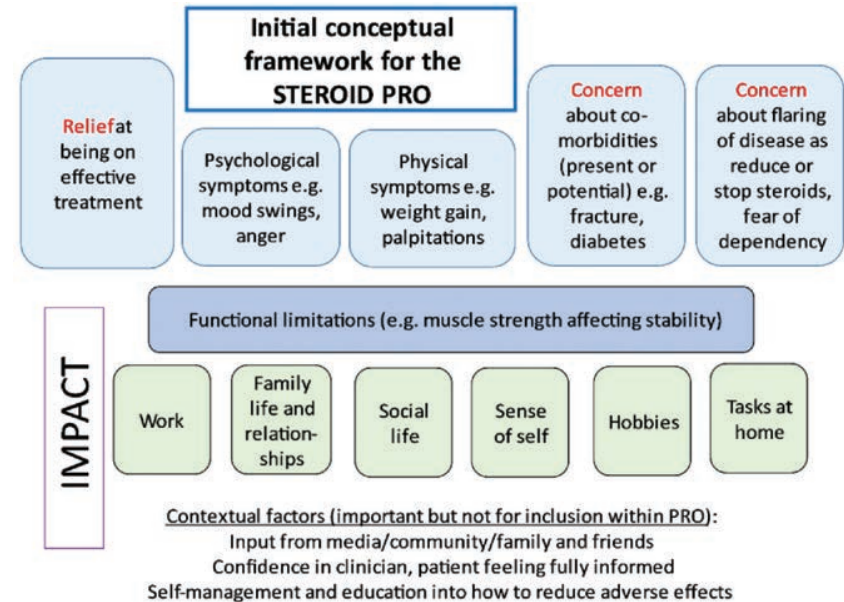

Figure 1.

glucocorticoid therapy. Future work will include inductive analysis of qualitative transcripts to inform candidate questionnaire items, cognitive interviewing linguistic translatability assessment, and an international validation survey to define the final PROM questionnaire and its measurement properties.

\section{REFERENCES:}

[1] Cheah JTL, Robson JC, Black RJ, et al. The patient's perspective of the adverse effects of glucocorticoid use: A systematic review of quantitative and qualitative studies. From an OMERACT working group. Semin Arthritis Rheum. 2020 Oct; 50(5):996-1005.

[2] Black RJ, Robson JC, Goodman SM, et al. A Patient-reported Outcome Measure for Effect of Glucocorticoid Therapy in Adults with Inflammatory Diseases Is Needed: Report from the OMERACT 2016 Special Interest Group. J Rheumatol. 2017; 44(11):1754-8.

[3] Cheah JTL, Black RJ, Robson JC, et al. Toward a Core Domain Set for Glucocorticoid Impact in Inflammatory Rheumatic Diseases: The OMERACT 2018 Glucocorticoid Impact Working Group. J Rheumatol. 2019; 46(9):1179-1182. Disclosure of Interests: Susan Bridgewater Grant/research support from: Grant from Vifor Pharma for an independent investigator-led study to develop a PRO for steroids, Jill Dawson: None declared, Mwidimi Ndosi: None declared, Rachel J Black: None declared, Jonathan T.L. Cheah: None declared, Emma Dures: None declared, Nilasha Ghosh: None declared, Elizabeth A Hoon: None declared, Iris Navarro-Millan Consultant of: Received consultant fees from SOBI, Diyu PearceFisher: None declared, Pamela Richards: None declared, Carlee Ruediger: None declared, Christine Silverthorne: None declared, Joanna Tieu Grant/research support from: Vifor Pharma, Sarah Mackie Consultant of: Consultancy on behalf of institution for Roche/Chugai, Sanofi, AbbVie and AstraZeneca, Grant/research support from: Educational grant from Roche to attend EULAR2019, Susan Goodman: None declared, Catherine Hill: None declared, Joanna Robson Speakers bureau: Vifor Pharma for educational webinar, Grant/research support from: Grant from Vifor Pharma for an independent investigator-led study to develop a PRO for steroids

DOI: 10.1136/annrheumdis-2021-eular.164

\section{$\mathrm{AB} 0835$ \\ IS BASELINE VITAMIN D STATUS RELATED WITH THE RESPONSE TO BDMARDS IN SPONDYLOARTHRITIS} PATIENTS?

B. M. Fernandes ${ }^{1}$, S. Garcia ${ }^{1}$, F. Oliveira Pinheiro ${ }^{1}$, M. Rato ${ }^{1}$, D. Fonseca ${ }^{2}$, D. Santos Oliveira ${ }^{1,3}$, A. Martins ${ }^{1}$, F. R. Martins ${ }^{4}$, M. Bernardes $^{1,5}$, L. Costa $^{1}$ ${ }^{1}$ Centro Hospitalar Universitário São João, Rheumatology, Porto, Portugal; ${ }^{2}$ Centro Hospitalar Vila Nova de Gaia/Espinho, Rheumatology, Vila Nova de Gaia, Portugal; ${ }^{3}$ Faculty of Medicine, University of Porto, Center for Health Technology and Services Research (CINTESIS), Porto, Portugal; ${ }^{4}$ Centro Hospitalar Universitário do Algarve, Rheumatology, Faro, Portugal; ${ }^{5}$ Faculty of Medicine, University of Porto, Departamento de Medicina, Porto, Portugal

Background: Vitamin $D$ is thought to have an important role in immune regulation and is being subject of research in several autoimmune diseases. Some data suggest that vitamin D deficiency is common in Spondyloarthritis ( $\mathrm{SpA}$ ) and may be associated with disease activity and structural damage.

Objectives: To evaluate if there is a relation between baseline vitamin $D$ status and the response to biologic disease-modifying antirheumatic drugs (bDMARDs) in a SpA monocentric cohort.

Methods: Retrospective study including all the SpA patients (ASAS classification criteria) followed at our Rheumatology Department, registered in the 